Japines Prychologial Revearich

1994. Vol 36, No. 4, 211.218

\title{
Involuntary attention driven by abrupt change in early visual features
}

\author{
HIROMI MORITA \\ Deparment of Pswchology, Faculty of Literature, University of Tokvo: Bunkvo-ku, Tokyo 113
}

I found that change in early visual features generally evokes involuntary attention, which is called stimulus-driven attention. In Experiment 1, luminance was increased (light condition) or decreased (dim condition) in the periphery as a cue. Then the subjects judged which of the two light spots. one on the cued side and the other on the opposite side, was presented earlier. Although the spots were presented simultaneously at an equal eccentricity, consistent responses (responses to the cued side) were significantly more frequent than inconsistent ones immediately after the presentation of the cue. This result indicates that stimulus-driven attention affects temporal-order judgments. In Experiment 2, the cue was a change in color (color condition) or orientation (orientation condition) in the periphery. Consistent responses were also more frequent, which means that color and orientation evoke stimulus-driven attention. These results can be explained by a model that a single mechanism of attention is driven by early visual processing modules

Key words: attention. stimulus-driven attention, features, temporal-order judgments, early visual processing modules.

If one's attention is focussed to a part of the visual field, speed and accuracy of his response to stimuli are enhanced in that region. This is the effect of spatial attention (Eriksen \& St. James, 1986; Eriksen \& Yeh, 1985; LaBerge. 1983; LaBerge \& Brown, 1989; Posner, Snyder, \& Davidson, 1980; Shaw, 1978).

One typical example of the spatial attention effect is seen in the following simple detection task: The reaction time to a small light spot is shortened if it appears at the position to which attention is directed (Posner et al,, 1980; Posner, 1980). It is

' The author would like to thank Professor Kazuo Nakatani and Professor Shinsuke Shimojo for their guidance throughout this study. The author also thanks Professor Kouichi Oda for lending a device for the experiments and Professor Masahiko Morita for helpful advice.

${ }^{2}$ Now at Perception Laboratory, Human Informatics Department, National Institute of Biosciences and Human-Technology, 1-1, Higashi, Tsukuba, Ibaraki 305. thought that this is because voluntary attention promotes the transmission speed of the information in the visual pathway.

Recently it has been reported, however, that the attention effect can be evoked, without voluntary control of attention, by a stimulus itself: Posner and Cohen (1984) brightened or dimmed a peripheral stimulus irrelevant to the task, and found a transient attention effect around it.

Yantis and Jonides (1984) showed that the abrupt onset of a stimulus captures attention. They investigated the attention effect using visual search tasks and obtained the following result. When there is an abrupt onset of the target in the search display, it is easy to detect; when there is an abrupt onset of the distractor, a large interference effect is observed. They concluded that the stimulus with abrupt onset captures attention when the subject does not focus his attention anywhere else (Yantis \& Jonides, 1990).

These attention effects evoked by external stimuli have been called the effects of "automatic allocation of attention" (Yantis \& 
Jonides, 1990) or "exogenous attention" (Allport, 1989). However, I will call them the effects of "stimulus-driven attention" in the present paper because I want to emphasize that these effects are caused by external stimuli.

The visual information processing system contains several processing modules each of which detects an early visual feature such as luminance, color and orientation. It is thought that by directing voluntary attention to a location, the outputs of these modules are integrated and attributed to an object. Then how does the mechanism of stimulusdriven attention affect the visual information processing system? Does it work at the stage of integration of features, or at the stage of detection of a feature in a module?

One approach to this problem is to examine the effect of attention driven by a feature (e.g. color) on a module which processes another feature (e.g. luminance module). If the mechanism of stimulus-driven attention works within a module, such inter-module effect will not be observed. However, only attention effect driven by luminance change on luminance detection has been reported so far (Jonides \& Yantis, 1988).

This study aims to clarify what stage the mechanism of stimulus-driven attention works at. For this purpose, I examined whether luminance detection is influenced by change in color or orientation.

In order to examine the attention effect on luminance detection tasks, I used a temporalorder judgment task instead of reaction time measurement. This task was also used in the work of Stelmach and Herdman (1991). They presented two light spots simultaneously in both sides of the fixation point at an equal eccentricity; the subject perceived earlier the spot at the position to which he had directed attention voluntarily in advance. Thus we can find the attention effects by measuring this subjective temporal order.
This method enables us to detect slight effects of attention because the subjective temporal order is hardly affected by such noises that originate from the motor pathway, unlike the reaction time measurement.

In the following experiments, first I will show that the subjective temporal order is caused by stimulus-driven as well as voluntary attention. Then I will show that the attention effect is evoked by change in color or orientation, without change in luminance, using the temporal-order judgment task.

\section{Experiment 1}

The purpose of Experiment 1 was to examine whether subjective temporal order is caused by stimulus-driven attention.

The cue ${ }^{3}$ which was used to evoke attention effects was based on that used by Posner and Cohen (1984). They presented two square frames on both sides of the fixation item. Then they brightened or dimmed one of the frames which evoked an attention effect. The effect was, however, rather small.

In the present experiment, I caused a luminance change and an abrupt appearance of a pattern to occur simultaneously in order to obtain a larger attention effect. Concretely, a square frame was presented in the periphery by changing luminance in the corresponding region: The condition in which the frame was presented by lightening is called "the light condition"; the condition in which it was presented by dimming is called "the dim condition."

After some time has passed (a cue lead time) from the presentation of the square

"It should be noted that the term "cue" is used to represent the stimulus (or the change in stimulus) presented to draw stimulus-driven attention as well as voluntary attention. It is not necessarily relevant to the task. 
frame, two light spots were presented simultaneously: One at the center of the frame and the other at the opposite position as to the fixation point. These spots were the probes used in order to measure subjective temporal order.

Method

Subjects. Six undergraduate students participated in the experiment. They were unaware of the purpose of the experiment.

Stimuli and apparatus. The experiment was conducted in a light room. The stimuli were presented on a CRT display (SANYO CMT-A 14HU) controlled by a personal computer (NEC PC-9801DA). A chin rest was used to maintain the viewing distance of 57 $\mathrm{cm}$.

Three types of display were used (Figure 1): fixation display. cue display, and probe display. The intensity in the display was different for the light condition and the dim condition.

The fixation display contained only a white fixation point $10.1 \times 0.1 \mathrm{deg} ; 62.5$ $\mathrm{cd} / \mathrm{m}^{2}$ against a uniform green background $\left(25.0 \times 15.5 \mathrm{deg}: 0.3 \mathrm{~cd} / \mathrm{m}^{2}\right.$ in the light con- dition, $1.4 \mathrm{~cd} / \mathrm{m}^{2}$ in the dim condition).

The cue display contained a square frame, which was different from the background in luminance, superimposed on the fixation display: The center of the frame was $3.9 \mathrm{deg}$ above the fixation point and $5.0 \mathrm{deg}$ to the left or right of the fixation point. The outside diameter was $4.2 \mathrm{deg}$ and the inside diameter was $2.5 \mathrm{deg}$. The intensity was $1.4 \mathrm{~cd} / \mathrm{m}^{2}$ in the light condition and $0.3 \mathrm{~cd} / \mathrm{m}^{2}$ in the $\mathrm{dim}$ condition.

The probe display contained two white spots $\left(0.3 \times 0.3 \mathrm{deg} ; 62.5 \mathrm{~cd} / \mathrm{m}^{2}\right)$ (probes) superimposed on the cue display: One was at the center of the square frame, and the other was at the same position but on the opposite side relative to the fixation point.

Procedure. Each trial began with the presentation of the fixation display for 708 , 885,1062 , or $1239 \mathrm{~ms}$, followed by the cue display. The cue display was presented for $53,106,212,425$, or $850 \mathrm{~ms}$ (cue lead time), and was replaced by the probe display.

Subjects were then required to judge which of the probes, left or right, appeared first and to make a response by pressing the

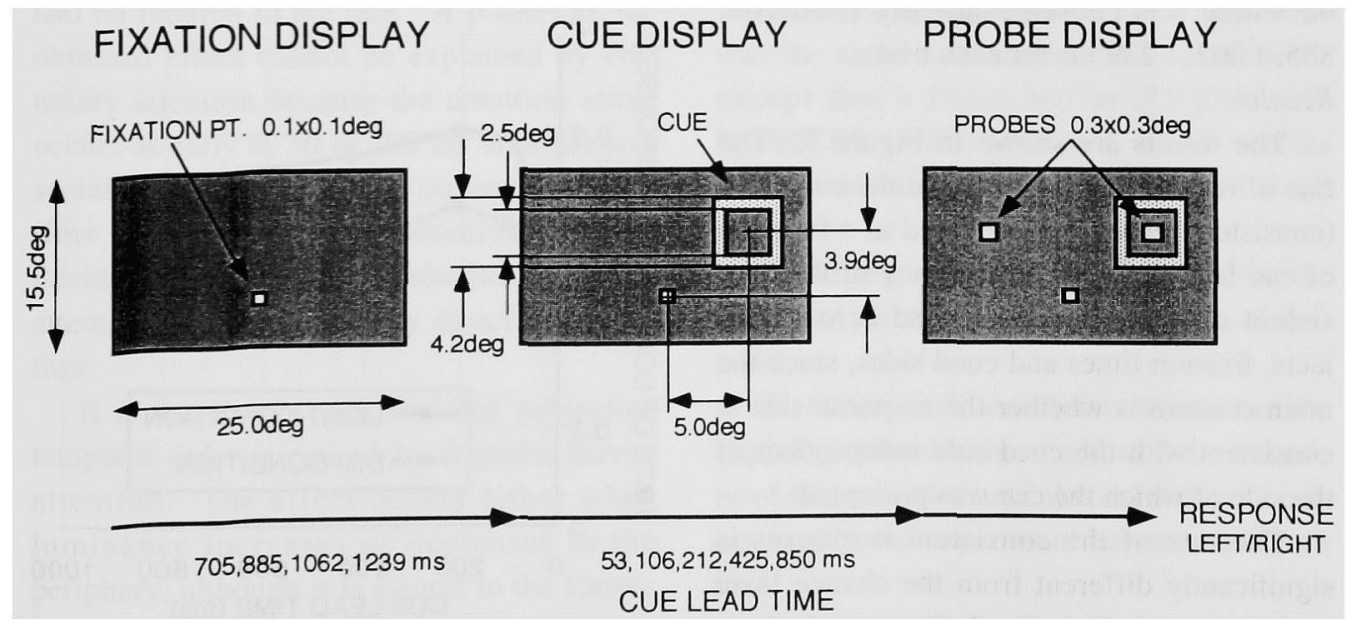

Figure 1. Schematic representation of the display used in Experiment 1: In the light condition, first, the fixation point is presented in a dark green background (fixation display). Then, a square frame of light green color appears on the left or right side (cue display). After a cue lead time, two probes are presented on both sides (probe display). 
response key corresponding to the probe. As soon as the subjects made their responses, the next trial began.

The subjects had been told to keep fixating the central spot during each trial. With regard to the cue, they had been told that "a square will appear on the display during the trial, but it has no relation to the task."

Before beginning the experiment, the experimenter adjusted the horizontal position of the fixation point until the subject perceived the two probes appearing simultaneously when there was no cue.

Then, after being given the instruction and practicing 10 or 20 trials, the subject started the actual experiments.

Design. Each subject participated in two sessions: the light condition and the dim condition. The order of the sessions was counterbalanced across the subjects.

There were 10 conditions in each session, which were defined by the factorial combination of two loci of the cue (left and right) and five cue lead times $(53,106,212,425$, and $850 \mathrm{~ms}$ ). There were 200 trials, 20 trials for each condition randomly mixed in a session. The fixation display was presented for a period which was chosen randomly from 708 , $885,1062,1239 \mathrm{~ms}$ for each trial.

Results

The results are shown in Figure 2. The rate of response to the probe in the cued side (consistent responses) is plotted as a function of cue lead time. The frequency of the consistent responses was summed across subjects, fixation times and cued sides, since the main concern is whether the response side is consistent with the cued side independent of the side of which the cue was presented.

The rate of the consistent responses is significantly different from the chance level $(0.5)$ in both light and dim conditions $\left(\chi^{2}=310.2, d f=5, p<0.01\right.$ in the light condition; $\chi^{2}=43.1, d f=5, p<0.01$ in the dim condition). The rates are large especially at short cue lead times.

The difference in rates of consistent responses between the light condition and the dim condition is also significant $\left(\chi^{2}=86.1\right.$, $d f=5, p<0.01$ ). At all cue lead times the rates of the consistent responses in the light condition are larger than those in the dim condition.

There is a significant time dependence of the rate of the consistent responses in both conditions $\left(\chi^{2}=13.6, d f=4, p<0.02\right.$ in the light condition; $\chi^{2}=18.2, d f=4, p<0.01$ in the $\operatorname{dim}$ condition). Functions for both conditions rise until $212 \mathrm{~ms}$, then that for the dim condition falls below the chance level at $850 \mathrm{~ms}$, while that for the light condition remains above the chance level.

\section{Discussion}

The result that the subject made consistent responses more frequently than inconsistent ones indicates that he has perceived the spot presented at the center of the frame earlier than the other. Another interpretation is that the subject might have perceived the two probes simultaneously in every trial, but made a response blindly to the cued side,

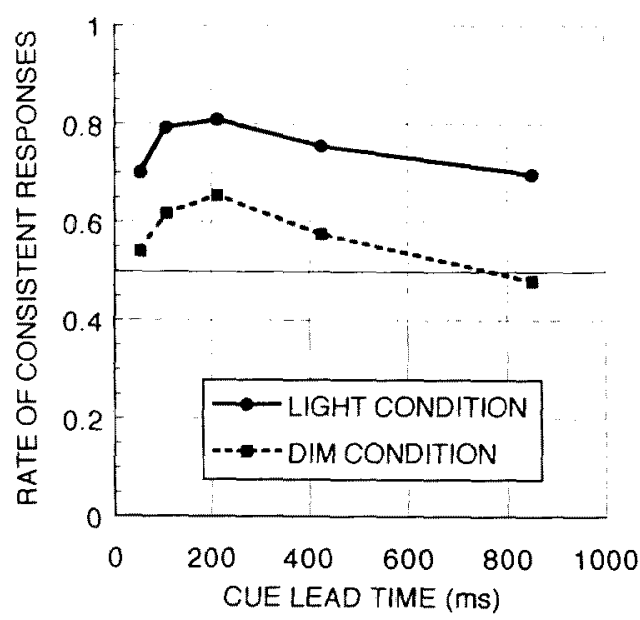

Figure 2. Rate of consistent responses as a function of cue lead time in Experiment 1. The responses to the cued side were summed across six subjects and two sides of the cue presentation. 
since he found no difference between the two probes except that one of them was found within the frame. This latter idea, however, does not explain why the rate of consistent responses depends on cue lead time. Moreover. most of the subjects reported after the experiment that they had often perceived two spots appearing in sequence clearly. Thus it is considered that the result reflects subjective temporal order.

Preceding luminance change also causes a forward masking effect on detection of the following light spot (Breitmeyer, 1984). In the present experiment, however, the masking effect was negligible, since the difference in intensity between the light spots and the cue was large enough. Moreover, the masking effect prevents the detection of the light spot in the frame. Thus the result cannot be explained by the masking effect.

Therefore, the subjective temporal order is attributed to directed attention. This attention is not voluntary but is driven by stimulus for the following reasons. Operationally, directing attention to the cue was not advantageous in performing the task, and the subjects were instructed that the square frame had no relation to the task. A posteriori, the obtained effect cannot be explained by voluntary attention because the attention effect occurs as early as 50 or $100 \mathrm{~ms}$ after the presentation of the cue, which seems too short a time for the subject to perceive the cue, decide on which side he should direct his attention to, and to actually direct the attention.

It is therefore concluded that subjective temporal order is caused by stimulus-driven attention. The effect occurs either when luminance increases or decreases in the periphery, although it is clearer in the former case.

The temporal characteristics of the attention effect, which increases before $200 \mathrm{~ms}$ and decreases after that, resemble those obtained in Posner and Cohen (1984) and Nakayama and Mackeben (1989).

In the light condition, the attention effect still remained at $850 \mathrm{~ms}$ whereas it did not in the dim condition. This offset can be attributed to the existence of the square frame which has higher luminance than the background; according to Hikosaka, Miyauchi, and Shimojo (1993), such stimuli of higher luminance cause sustained attention effect.

\section{Experiment 2}

Since it has been indicated in Experiment 1 that subjective temporal order is produced by stimulus-driven attention, temporal-order judgment tasks are again used in Experiment 2 in order to examine whether stimulus-driven attention is evoked by change in color or orientation. If it were evoked, the subjective temporal order would be observed in the same manner as in Experiment 1.

\section{Method}

Subjects. Seven undergraduate students participated in the experiment. They did not participate in Experiment 1 and were unaware of the purpose of the experiment.

Stimuli and apparatus. The apparatus was the same as that used in Experiment 1 except that a frame buffer (Digital Arts HyPER-FRAME+) was used, which enables controls of intensity of the display in small steps.

The three types of display used in the color and orientation conditions were as follows.

1. Color condition: Before the start of the experiment, each subject adjusted the intensity of the red color until the red and gray colors used in the experiment became equiluminant.

The fixation display contained only a white fixation point $(0.1 \times 0.1 \mathrm{deg} ; 62.5$ $\mathrm{cd} / \mathrm{m}^{2}$ against a uniform gray background $\left(25.0 \times 15.5 \mathrm{deg} ; 3.3 \mathrm{~cd} / \mathrm{m}^{2}\right)$. 
The cue display contained a red square superimposed on the fixation display: The square and the background were equiluminant. The center of the square was $3.9 \mathrm{deg}$ above the fixation point and $5.0 \mathrm{deg}$ to the left or right of the fixation point. The side was 4.2 deg long.

I used the filled square as a cue which is expected to attract larger attention than the square frame used in Experiment 1; note that presentation of the cue does not cause a change in luminance contrast between the probe and its surrounding in this experiment.

The probe display contained two white spots $\left(0.3 \times 0.3 \mathrm{deg} ; 62.5 \mathrm{~cd} / \mathrm{m}^{2}\right)$ (probes) superimposed on the cue display: One was at the center of the square, and the other was at the opposite position as to the fixation point.

2. Orientation condition: The fixation display contained only a white fixation point $\left(0.1 \times 0.1 \mathrm{deg} ; 62.5 \mathrm{~cd} / \mathrm{m}^{2}\right)$ against a background $(25.0 \times 15.5 \mathrm{deg})$. The background was made of $80 \times 50$ small green bars $(0.04 \times$ $0.3 \mathrm{deg} ; 21.8 \mathrm{~cd} / \mathrm{m}^{2}$ ) with a uniform orientation (45 deg to the right from the vertical).

The cue display contained a square which was made of $8 \times 8$ bars with a different orientation from the background $(45 \mathrm{deg}$ to the left from the vertical). The position and the size of the square were the same as those in the color condition.

The probe display contained probes having the same properties as those in the color condition superimposed on the cue display.

For half of the subjects, the orientation of the bars which constituted the square and the background was changed.

Procedure. The temporal sequence of the stimuli was the same as in Experiment 1 except that the cue lead time of $-106 \mathrm{~ms}$ was used instead of $53 \mathrm{~ms}$. Following the presentation of the fixation display, the probes were introduced on the display; after $106 \mathrm{~ms}$ the cue was superimposed.This condition was employed to check on the subjects who responded blindly to the cued side.

Design. Each subject participated in two sessions: the color condition and the orientation condition. The order of the sessions was counterbalanced across the subjects.

There were 10 conditions in each session, which were defined by the factorial combination of two loci of the cue (left and right) and five cue lead times $(-106,106,212,425$, and $850 \mathrm{~ms}$ ). There were 200 trials, 20 trials for each condition randomly mixed in a session. The fixation display was presented for a period which was chosen randomly from $708,885,1062,1239 \mathrm{~ms}$ for each trial.

Results

The results are shown in Figure 3. As in Experiment 1, the rate of consistent response (summed across subjects and cued side) is plotted as a function of cue lead time.

The rate of the consistent responses is significantly different from the chance level in both color and orientation conditions $\left(\chi^{2}=50.9, d f=5, p<0.01\right.$ in the color condition; $\chi^{2}=73.8, d f=5, p<0.01$ in the orientation condition). The rates are large especially at small cue lead times.

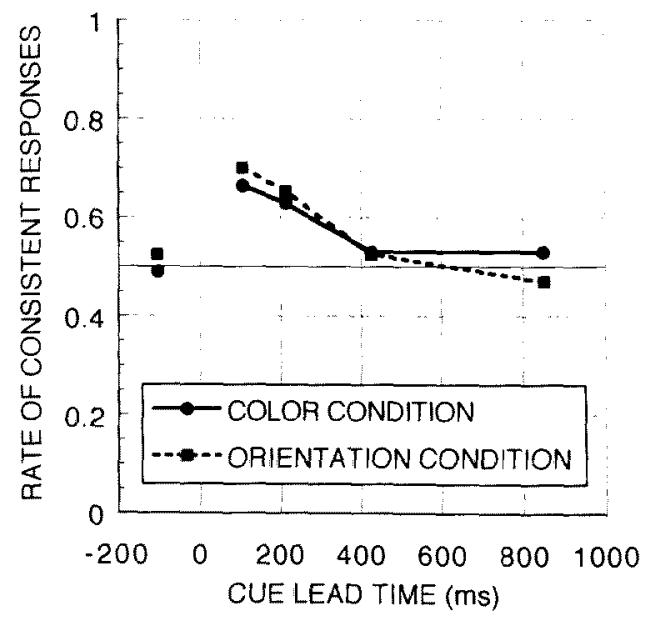

Figure 3. Rate of consistent responses as a function of cue lead time in Experiment 2. The responses to the cued side were summed across seven subjects and two sides of the cue presentation. 
However, at the cue lead time of -106 $\mathrm{ms}$, the rate of the consistent responses is near the chance level in both conditions $(\chi=0.129, d f=1, p>0.1$ in the color condition: $\chi=0.7, d f=1, p>0.1$ in the orientation condition). which indicates that the subjects did not respond to the cued side blindly.

There is a significant time dependence of the rate of the consistent responses in both conditions at the cue lead times except for $-106 \mathrm{~ms}(\chi=16.3, d f=3, p<0.01$ in the color condition: $\chi=40.7, d f=3, p<0.01$ in the orientation condition). Both functions have a large value at $106 \mathrm{~ms}$; however, that of the orientation condition falls below the chance level at $425 \mathrm{~ms}$.

The difference in rates of consistent responses between the color condition and the orientation condition is not significant $\left(\chi^{2}=4.0, d f=5, p>0.1\right)$. The results obtained for the two conditions are very similar.

Discussion

The result shows that the subject perceived the probe presented in the cue earlier than the other probe. It is clear that the subject did not respond blindly to the cued side, because in the condition of the cue lead time of $-106 \mathrm{~ms}$ the rate of consistent responses is at the chance level. The subjective temporal order obtained here can be attributed to stimulus-driven attention, just as in Experiment 1. It is thus concluded that stimulus-driven attention is caused by the change in color and orientation.

The temporal characteristics of the attention effect in the color and orientation conditions are almost the same in that the effect decreases after $106 \mathrm{~ms}$ and has no offset.

\section{General Discussion and Conclusions}

The results of Experiment 2 show that luminance detection is affected by the outputs of not only the luminance-processing module but also the color- and orientation- processing modules. This indicates that the mechanism of stimulus-driven attention works not within a module, but across modules.

Another important point is that temporal characteristics of the attention effects are quite similar between the color and orientation conditions. Moreover, they also resemble the temporal characteristics obtained in the dim condition in Experiment 1.

These results can be explained by the use of two models. One is that each processing module has its own attention mechanism, which is driven selectively by the corresponding feature and can affect the processing of other features. This model seems unnatural, however, in that the attention mechanisms of all modules are required to affect luminance detection with the same temporal characteristics. The other model is that a single mechanism is driven by the output of various modules and affects the processing of various modules. This model is simpler and naturally leads to the above agreement on the temporal characteristics.

Based on the second model, the effect of stimulus-driven attention can be described as follows: The mechanism of stimulus-driven attention receives input separately from the modules processing luminance, color, and orientation. Change in whatever feature (luminance, color, orientation, etc.) is detected by one of the modules corresponding to the feature, and the module drives the same mechanism. Then the output of the mechanism gives facilitation to somewhere in the visual pathway (in this case, the luminancedetection pathway).

The result that attention was driven by color and orientation even in luminance detection tasks may appear to be inconsistent with the "contingent involuntary orienting hypothesis" put forward by Folk, Remington, and Johnston (1992). According to their hypothesis, involuntary attention is evoked 
only by the stimulus which shares the critical property for performing the task. The critical properties they dealt with, however, are "dynamic discontinuities" (whether the stimulus changes temporally) and "static discontinuities" (whether the stimulus is spatially distinct from others). Although the task and the cue in the present paper use different features, they are both of dynamic discontinuities. The obtained result is not, therefore, inconsistent with the above hypothesis; it rather supplements the hypothesis by showing that the features used in the task and the cue need not be the same, as long as they are of dynamic discontinuities, for stimulus-driven attention.

In conclusion, I have shown that the change in color or orientation evokes stimulus-driven attention in the same manner as the change in luminance. I also presented a model that there exists a single mechanism of attention driven by output of various early visual processing modules. This model can explain the experimental results simpler than other models, although it has not been proven yer.

More experimental evidence supporting this model is required. Examination of the influence of voluntary control on the attention mechanism in this model also remains for future studies.

\section{References}

Allport, A. 1989 Visual attention. In M. I. Posner (Ed.), Foundations of cognitive science. Cambridge, MA: MIIT Press. Pp. 631-681

Breitmeyer. B. G. 1984 Visual masking: An integrative approach (Oxford psychology series No. 4). Oxford: Oxford University Press.

Eriksen, C. W., \& St. James, J. D. 1986 Visual attention within and around the field of focal attention: A zoom lens model. Perception \& Psychophysics, 40, 225-240.

Eriksen, C. W., \& Yeh, Y. 1985 Allocation of attention in the visual field. Journal of Experimental Psychology: Human Perception and
Performance, 11, 583-597.

Folk, C. L., Remington, R. W., \& Johnston, J. C. 1992 Involuntary covert orienting is contingent on attentional control settings. Journal of Experimental Psychology: Human Perception and Performance, 18, 1030-1044.

Hikosaka, O., Miyauchi, S., \& Shimojo, S. 1993 Focal visual attention produces illusory temporal order and motion sensation. Vision Research, 33, 1219-1240.

Jonides, J., \& Yantis, S. 1988 Uniqueness of abrupt visual onset in capturing attention. Perception $\&$ Psychophysics, 43, 346-354.

LaBerge, D. 1983 The spatial extent of attention to letters and words. Journal of Experimental Psychology: Human Perception and Performance, 9, $371-379$.

LaBerge, D., \& Brown, V. 1989 Theory of attentional operations in shape identification. Psychological Review, 96, 101-124.

Nakayama, K., \& Mackeben, M. 1989 Sustained and transient components of focal visual attention. Vision Research, 29, 1631-1647.

Posner, M. I. 1980 Orienting of attention. Quarterly Journal of Experimental Psychology, 32, 3-25.

Posner, M. 1., \& Cohen, Y. 1984 Components of visual orienting. In H. Bouma \& D. G. Bouwhuis (Eds.), Attention and performance $X$. Hillsdale, NJ: Lawrence Erlbaum Associates. Pp. 531-556.

Posner, M. I., Snyder, C. R. R., \& Davidson, B. J. 1980 Attention and the detection of signals. Joumal of Experimental Psychology: General. 109, 160 - 174 .

Shaw, M. L. 1978 A capacity allocation model for reaction time. Journal of Experimental Psychology: Human Perception and Performance, 4, 586-598.

Stelmach, L. B., \& Herdman, C. M. 1991 Directed attention and perception of temporal order. Journal of Experimental Psychology: Human Perception and Performance, 17, 539-550.

Yantis, S., \& Jonides, J. 1984 Abrupt visual onsets and selective attention: Evidence from visual search. Journal of Experimental Psychology: Human Perception and Performance, 10, 601 621.

Yantis, S., \& Jonides, J. 1990 Abrupt visual onsets and selective attention: Voluntary versus automatic allocation. Journal of Experimental Psychology: Human Perception and Performance, 16, $121-134$.

(Received March 1, 1993; accepted March 5, 1994) 